\title{
Capability of ischemia-modified albumin to predict serious cardiac outcomes in the short term among patients with potential acute coronary syndrome
}

\author{
Andrew Worster, P.J. Devereaux, Diane Heels-Ansdell, Gordon H. Guyatt, John Opie, \\ Farouk Mookadam, Stephen A. Hill
}

ß See related article page 1697

\section{Abstract}

Background: Ischemia-modified albumin (IMA) has been suggested as a marker of cardiac ischemia. Little, however, is known about its capacity to predict short-term serious cardiac outcomes (death, myocardial infarction, congestive heart failure, serious arrhythmia, or refractory ischemic cardiac pain) in patients arriving at the emergency department with symptoms that may indicate cardiac ischemia.

Methods: We screened 546 patients over a 4-week period, of whom 189 fulfilled our entry criteria by presenting to an emergency department with potential cardiac-ischemia symptoms within 6 hours after chest pain, seeing an emergency physician who chose to order a troponin I test, and having no serious cardiac outcome before the troponin result became available. We followed the study patients for 72 hours to determine if any experienced a serious cardiac outcome. We calculated the likelihood ratios (LRs) of IMA findings predicting serious cardiac outcomes that could not be diagnosed at presentation with current techniques.

Results: Of the 189 patients, 24 had a serious cardiac outcome within 72 hours after their arrival at the emergency department. The likelihood ratios for IMA measurement within 6 hours after chest pain predicting a serious cardiac outcome within the next 72 hours were 1.35 (95\% confidence interval [Cl] 0.315-5.79) for IMA $\leq 80 \mathrm{U} / \mathrm{mL}$ and $0.98(95 \% \mathrm{Cl} 0.86-$ 1.11) for $I M A>80 \mathrm{U} / \mathrm{mL}$.

Conclusions: These data suggest that in patients presenting with chest pain who have not yet experienced a serious cardiac event, IMA is a poor predictor of serious cardiac outcomes in the short term.

CMAJ 2005;172(13):1685-90

$\mathrm{P}$ atients who arrive at an emergency department with potential cardiac symptoms present a diagnostic challenge to physicians, who must also decide resource utilization within the medical system. Physicians are under pressure to minimize resource use and to stream patients into a level of care appropriate to their evolving clinical condition. A method to predict the likelihood of a serious cardiac outcome (death, myocardial infarction [MI], congestive heart failure, serious arrhythmia or refractory ischemic cardiac pain) during the next 72 hours would therefore be very advantageous. Appropriate management of patients whose condition cannot be diagnosed at presentation (and who therefore cannot immediately be streamed into an appropriate level of care) is uncertain. The role of cardiac biochemical markers in risk prediction for this group of patients remains unclear.

Alteration of human serum albumin by ischemia has recently been evaluated as a serum biomarker of cardiac ischemia. ${ }^{1}$ The amino terminal end (N-terminal) of the albumin molecule is a binding site for transitional metals such as cobalt, copper and nickel. ${ }^{1}$ Possibly as the result of hypoxia, acidosis, free-radical injury and energydependent membrane disruption, the $\mathrm{N}$-terminal undergoes a decrease in binding capacity in the presence of ischemia. ${ }^{2-4}$ This alteration can be measured: a set amount of cobalt is added to the patient's serum, after which a colorimetric assay, the albumin-cobalt binding assay, is used to determine the amount of cobalt that remains unbound. An elevated concentration of ischemia-modified albumin (IMA) has, therefore, been proposed as a marker of myocardial ischemic injury. ${ }^{4-6}$ This assay is reported to be positive within minutes of ischemia and remains so for up to several hours later, allowing detection before the development of myocardial necrosis (as evidenced by normal levels of creatinine kinase isoenzyme [CK-MB], troponin and myoglobin). ${ }^{5,7}$

The ability to detect ischemia before myocyte destruction would allow for earlier and more accurate management decisions for patients suspected of having acute coronary syndrome (ACS) than is currently possible by measuring serum troponin, $\mathrm{CK}-\mathrm{MB}$ or myoglobin levels. A test that detects all ongoing ischemia that could ultimately cause adverse events would also allow cardiac ischemia to be ruled out, permitting earlier discharge and fewer unnecessary investigations. Because of its potential capacity to identify acute cardiac ischemia, IMA measurement has been proposed as a means to triage patients arriving with symptoms that might result from cardiac ischemia. ${ }^{8}$ This 
study sought to determine the capacity of IMA testing to predict serious cardiac outcomes in the short term, in patients presenting to the emergency department with potential cardiac ischemia symptoms.

A study of a diagnostic test is valid insofar as the investigators enrol an appropriate sample of patients and conduct an independent, blinded comparison with the method currently accepted as standard. ${ }^{9}$ Those in whom the diagnosis is in doubt represent an appropriate sample of patients. Patients presenting with an obvious disease are likely to have a different distribution of test results than those in whom the diagnosis is uncertain. Including such patients will therefore produce a biased - and likely, excessively sanguine - picture of the usefulness of the test under investigation.

In this case, we were interested in detecting ischemia that leads to death, MI, heart failure, serious arrhythmia or refractory pain during the next 72 hours. Our target population was patients at risk of such adverse events. Patients who arrive in the emergency department having had any of the target events, or who have one before the test result is available, do not need further diagnostic evaluation for their likelihood of subsequently having the event of interest. Furthermore, the distribution of test results from such people is likely to differ from that of patients who remain at risk. Our target sample, therefore, was those patients who remained at risk of adverse events when their first IMA test result was available. Our target finding was the incidence of any of the events of interest, determined by blinded adjudication conducted in duplicate.

\section{Methods}

Over 4 consecutive weeks in September 2003, we evaluated patients who arrived at the emergency department of the Hamilton General site of The Hamilton Health Sciences, a tertiary care centre staffed 24 hours a day by full-time emergency physicians. Eligible patients included all those aged 18 years or older with potential cardiac ischemia symptoms that occurred within 6 hours before their arrival at the emergency department, in whom the emergency physician elected to order a test for serum concentration of cardiac troponin I (cTnI). Participating patients were required to provide reliable contact information. We excluded patients referred directly to trauma or surgery; those in whom clinicians documented any of the study outcomes (death, MI, congestive heart failure, serious arrhythmia or refractory ischemic cardiac pain) before the results of their first cTnI becoming available; and patients who refused study participation.

Each participant's informed consent, demographic data, symptom characteristics and timing, and cardiac-related medical history were obtained by a research nurse. After a patient consented, blood for IMA measurements was drawn in the emergency department (called time 0) and again 3 and 6 hours later if the patient was not discharged in the meantime. Blood for cTnI levels was also drawn at time 0 , and subsequently at the discretion of the emergency physician.

We followed our study patients for 72 hours and recorded their status (alive, dead), location (inpatient, home, returned to hospital), need for therapeutic intervention and whether they had a serious cardiac outcome, defined as any of the following:

- Death, either cardiovascular (from a cardiovascular cause, including a revascularization procedure, cardiac arrest, MI, stroke or unknown cause) or noncardiovascular (from a clearly documented noncardiovascular cause such as infection, trauma or malignant disease).

- Myocardial infarction, as indicated by biochemical markers of myocardial necrosis (a typical rise and gradual fall of troponin concentrations or the more rapid rise and fall of CK-MB), along with at least 1 of the following: ischemic symptoms; development of pathologic Q waves on the electrocardiogram (ECG); electrocardiographic changes indicative of ischemia (elevation or depression of the ST segment); coronary artery intervention (e.g., coronary angioplasty); or pathologic findings of an acute MI.

- Congestive heart failure, as indicated by any 2 of these 3 criteria: chest radiograph consistent with congestive heart failure; clinical signs and symptoms consistent with heart failure; and/or administration of a diuretic.

- Serious arrhythmia (ventricular fibrillation, sustained ventricular tachycardia, asystole, electromechanical dissociation, supraventricular tachycardia, atrial fibrillation, sinus bradycardia, or second- or third-degree heart block), along with administration of cardioversion, atropine, an anti-arrhythmic, rate-slowing or sympathomimetic drug, or a temporary pacemaker.

- Refractory ischemic cardiac pain, defined as recurrent ischemic symptoms lasting 15 minutes or more, along with documented electrocardiographic changes indicative of ischemia or leading to a referral for an additional intervention such as thrombolytic therapy, cardiac catheterization or revascularization.

Two investigators (J.O. and P.J.D.) independently evaluated all cases of suspected serious cardiac outcomes while blinded to the IMA data. They resolved all disagreements by reaching a consensus opinion.

All blood samples for analysis were collected and transported immediately to the laboratory. Total CK, CK-MB and cTnI assays were done for all patients and the findings made available to clinical staff. Total CK levels were measured with the Roche Modular Analyzer (Roche Diagnostics, Laval, Que.). CK-MB mass and cTnI concentrations were measured with the Abbott AxSym (Abbott Laboratories Canada, Diagnostics Division, Mississauga, Ont.). These assays were done with reagents supplied by the manufacturers and carried out according to their respective protocols. Assay results are expressed as "units" per millilitre $(\mathrm{U} / \mathrm{mL})$, as defined by the developers; these are arbitrary units of absorbance derived from the calibration curve.

Red blood cells were immediately separated from serum for IMA analysis and frozen in liquid nitrogen for subsequent analysis. Samples were thawed in small batches; total time at room temperature from blood draw to analysis never exceeded 2 hours. IMA concentration was measured in the Roche Modular assay system by means of reagents, assay protocols and parameters from Ischemia Technologies (Denver, Colo.), in keeping with their recommended sample-handling procedures.

We calculated and have reported the sensitivities, specificities and likelihood ratios (LRs) with $95 \%$ confidence intervals (CIs) to describe the association with the occurrence of a serious cardiac outcome of IMA concentrations above or below a single threshold value of $80 \mathrm{U} / \mathrm{mL}$ for samples taken at time 0,3 hours, 6 hours and maximum IMA value. CIs for LRs were computed similarly 
to relative risk, by first calculating a variance and confidence limits for the logarithm of the LR, then transforming these limits to obtain a CI for the LR. CIs for sensitivity and specificity were calculated with the Wilson score method for binomial proportions. We explored the possibility of multiple thresholds or cut-off values, but the number of events proved too few. We examineded different thresholds, including $85 \mathrm{U} / \mathrm{mL}$ as suggested in the manufacturer's package insert, but no threshold produced appreciably different results than our initial choice of $80 \mathrm{U} / \mathrm{mL}$.

The Research Ethics Board of Hamilton Health Sciences and McMaster University approved the protocol.

\section{Results}

During the study period, 582 patients came to the emergency department with potential ACS symptoms, of whom 546 were screened (36 were missed; Fig. 1). Of these, 357 patients were excluded from participation, most (227) because they did not meet the inclusion criterion of ACS symptoms within the 6 hours before presentation at the emergency department (Table 1). Only 1 of the 189 enrolled patients could not be contacted directly by telephone 72 hours later. Research staff were able to confirm that the patient had not seen her family physician within 2 months after emergency department discharge. Moreover, her family physician had not received any notice of contact from any primary care practice or emergency department, and was able to confirm that the patient was still alive.

Differences in demographic and disease characteristics between the 24 patients who experienced a serious cardiac outcome and the 165 who did not were nonsignificant (Table 2). The 24 patients experienced a total of 35 cardiac outcomes (Table 3). Results for IMA analysis fell in a normal distribution from 57 to $138 \mathrm{U} / \mathrm{mL}$ (mean $92.3,95 \%$ CI 91.2-93.2, SD 11.5).

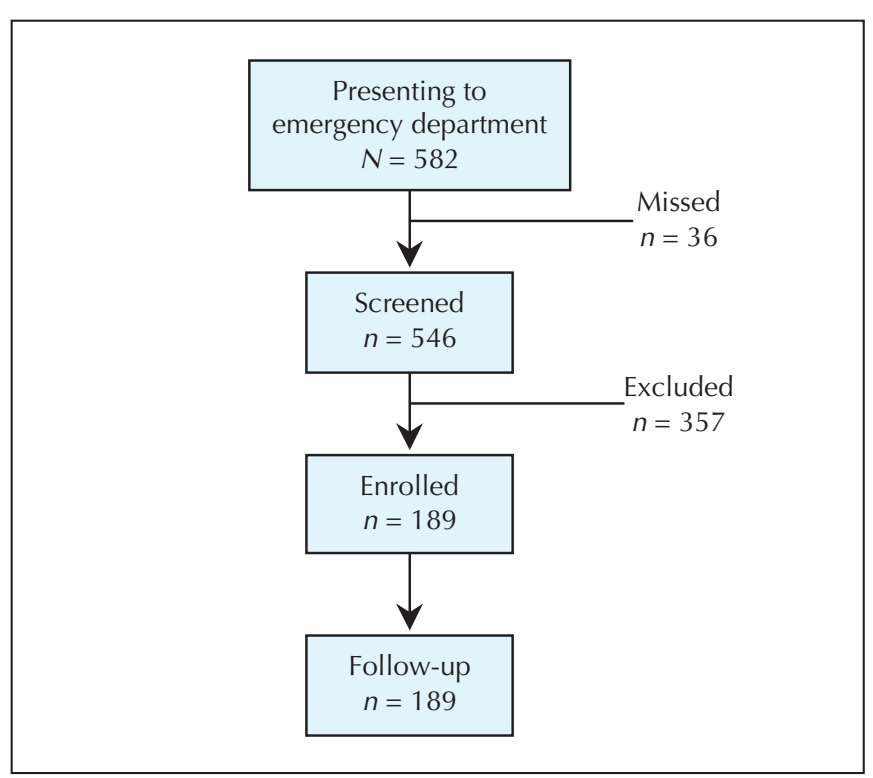

Fig. 1: Screening and enrolment of patients. Reasons for exclusion are listed in Table 1.
Estimates of diagnostic efficacy demonstrating the association of IMA levels above or below the $80 \mathrm{U} / \mathrm{mL}$ threshold value for the 3 times (time 0,3 and 6 hours) and the maximum value at any time revealed a maximum sensitivity of 92.3\% (95\% CI 66.7\%-98.6\%), found among patients with an IMA level above $80 \mathrm{U} / \mathrm{mL}$ when tested at 6 hours. The maximum specificity by IMA measurement was $24.3 \%$ (95\% CI 18.2\%-31.7\%), found among patients with serum IMA concentrations of $80 \mathrm{U} / \mathrm{mL}$ or less when tested at time 0 . In only 1 case did the $95 \%$ CIs of the corresponding LRs for each category exceed 10.0 or fall below 0.2 (for 6-hour negative LR, CI 0.17-10.03), and all cases included an LR value of 1.0 (Fig. 2).

\section{Interpretation}

This study revealed that the LRs associated with IMA levels in patients presenting to the emergency department with potential cardiac ischemia symptoms are close to 1 - so much so that they have minimal impact on the probability of short-term, serious cardiac outcomes. A limitation of our study was the size of the sample. This was designed as a pilot study, and one of our objectives was to estimate the sample size required for a larger trial. A larger sample would not only have improved the precision of our estimates, it would also have allowed us to apply multiple thresholds. However, our estimates of the LRs are so close to 1.0 , and the confidence intervals already sufficiently narrow, that it is unlikely that a larger study would demonstrate clinical usefulness for this test. A larger sample may have allowed us to perform post-hoc analysis to evaluate the usefulness of IMA measurement in a multiple-marker combination with other biochemical and clinical tools.

In this prospective study IMA results were not made available to physicians responsible for the care of the patient. Rather, we observed the ability of IMA testing to predict serious cardiac outcomes in our usual care model. A

Table 1: Reasons patients were excluded from participation in the study

\begin{tabular}{lc} 
& No. of patients \\
Criteria & $n=357$ \\
\hline
\end{tabular}

Inclusion criteria not met

Symptoms consistent with acute coronary syndrome in the 6 hours before presentation

Troponin I test ordered by the emergency physician

Patient able to provide a reliable contact

Patient consents to telephone contact for follow-up

Exclusion criteria met

Occurrence of an adverse event before the results of the troponin I test became available

Direct referral to trauma or surgery 
test that shows value in a prospective observational trial should then be evaluated in a prospective interventional trial, with test and control groups, to determine if serious cardiac outcomes can be attenuated or avoided with its use.

IMA analysis performed in real time would be available to the physician at about the same time as the troponin result. To examine the usefulness of the test in the situations when it is needed most - that is, when other clinical and laboratory tests are not diagnostic - we excluded all patients in whom a serious outcome could be diagnosed without the aid of laboratory testing, those admitted before troponin testing, those with diagnostic ECGs and those who suffered serous cardiac outcomes before the troponin result was available. ${ }^{10}$ Test characteristics are likely to differ in most of these situations because of greater ischemic damage. Thus, inclusion of such patients would give a misleading impression of IMA usefulness in the patients in whom clinicians would need the test to make a decision about hospital admission.

We chose to exclude patients whose chest pains began more than 6 hours before because several references indicate that IMA is rapidly cleared from the circulation after resolution of the period of ischemia and is unlikely to remain elevated longer than 6 hours. ${ }^{5,7}$

The strengths of this study include the careful selection

Table 2: Demographic and disease characteristics of enrolled patients

\begin{tabular}{|c|c|c|c|}
\hline \multirow[b]{2}{*}{ Characteristic } & \multicolumn{3}{|c|}{ Group; no. (\%) of patients* } \\
\hline & $\begin{array}{c}\text { All } \\
n=189\end{array}$ & $\begin{array}{c}\text { With an adverse } \\
\text { outcome } \\
n=24\end{array}$ & $\begin{array}{c}\text { Without } \\
\text { outcome } \\
n=165\end{array}$ \\
\hline Male & $112(59.3)$ & $16(66.7)$ & $96(58.2)$ \\
\hline Age, mean (SD), yr & $61.5(14.5)$ & $64.6(10.7)$ & $61.0(14.9)$ \\
\hline \multicolumn{4}{|l|}{ Presenting symptoms } \\
\hline Angina, chest pain & $166(87.8)$ & $22(91.7)$ & $144(87.3)$ \\
\hline Syncope/pre-syncope & $35(18.5)$ & $3(12.5)$ & $32(19.4)$ \\
\hline Palpitations & $21(11.1)$ & $4(16.7)$ & $17(10.3)$ \\
\hline Anginal equivalent & $123(65.1)$ & $15(62.5)$ & $108(65.5)$ \\
\hline Other pain & $12 \quad(6.3)$ & $2(8.3)$ & $10 \quad(6.1)$ \\
\hline Other discomfort & $24(12.7)$ & $2(8.3)$ & $22(13.3)$ \\
\hline \multicolumn{4}{|c|}{ Previous myocardial infarction } \\
\hline Yes & $56(29.6)$ & $9(37.5)$ & $47(28.5)$ \\
\hline No & $130(68.8)$ & $15(62.5)$ & $115(69.7)$ \\
\hline Unknown & $3(1.6)$ & $0 \quad(0.0)$ & $3(1.8)$ \\
\hline \multicolumn{4}{|c|}{ Tobacco smoking $(N=181)$} \\
\hline Current smoker & $44(24.3)$ & $3(13.6)$ & $41(25.8)$ \\
\hline Former smoker & $79(43.7)$ & $12(54.6)$ & $67(42.1)$ \\
\hline Never smoked & $58(32.0)$ & $7(31.8)$ & $51(32.1)$ \\
\hline \multicolumn{4}{|l|}{ Diabetes } \\
\hline Yes & $41(21.7)$ & $6(25.0)$ & $35(21.2)$ \\
\hline No & $147(77.8)$ & $18(75.0)$ & $129(78.2)$ \\
\hline Unknown & $1 \quad(0.5)$ & $0 \quad(0.0)$ & $1 \quad(0.6)$ \\
\hline
\end{tabular}

Note: All differences were statistically nonsignificant. $\mathrm{SD}=$ standard deviation. *Except as indicated for Age. of the patients for whom clinicians would be using IMA results to make decisions about hospital admission. Our standard for defining target-positive patients was highly patient-relevant: short-term, serious cardiac outcomes including death, MI, congestive heart failure, serious arrhythmia and refractory ischemic cardiac pain. In our evaluation of patients with suspected ACS, we used a 72-hour interval because events during this period dictate the need for immediate admission into the critical care unit. ${ }^{10} \mathrm{We}$ determined occurrence of target events through independent blind adjudication as well as successful follow-up of 188 (of 189) patients.

Since development of the albumin-cobalt binding assay, investigators have assessed IMA levels in a variety of clinical settings. Although cTnT measurements have been used to indicate that direct-current cardioversion (DCCV) does not cause cardiac damage, ${ }^{11}$ Roy and colleagues ${ }^{12}$ reported that in patients with atrial fibrillation, IMA levels are significantly elevated at 1 and 6 hours after DCCV, especially in patients with electrocardiographic evidence of post-DCCV ischemia. Sinha and associates ${ }^{13}$ evaluated ECG, cTnT and IMA levels individually and in combinations in patients diagnosed with non-ischemic chest pain, unstable angina and MI with ST- or non-ST-segment elevation. In this population, they found IMA measurements to be the most sensitive of 3 diagnostic tests and declared it to be highly sensitive for the diagnosis of myocardial ischemia in patients presenting with symptoms of ACS. ${ }^{13}$ Investigators have also reported IMA to be a sensitive measure of the magnitude and duration of ischemia induced during percutaneous coronary intervention $(\mathrm{PCI}){ }^{14,15} \mathrm{~A}$ subsequent PCI study supported this finding and also demonstrated a greater increase in IMA level from baseline in patients without collateral coronary-artery circulation than in those with it. ${ }^{16}$

A recent study ${ }^{17}$ involving patients with symptoms suggestive of ACS but with normal or nondiagnostic ECGs who came to the emergency department within 3 hours of chest pain documented outcomes at 30 days of ACS (unstable angina or MI with non-ST-segment elevation) or non-ischemic chest pain. They measured IMA at presentation and reported positive (LR 2.95, 95\% CI 1.91-4.56) and negative (LR $0.33,95 \%$ CI 0.21-0.52) likelihood ratios using a threshold of $93.5 \mathrm{U} / \mathrm{mL}$. From this, the inves-

\begin{tabular}{lc}
\hline \multicolumn{2}{l}{ Table 3: Breakdown of 35 serious } \\
cardiac outcome events in $\mathbf{2 4}$ patients \\
\hline Clinically serious event & $\begin{array}{r}\text { No. of } \\
\text { events }\end{array}$ \\
\hline Death & 0 \\
Myocardial infarction & 14 \\
Congestive heart failure & 5 \\
Clinically important arrhythmia & 3 \\
High-risk ischemia* & 13 \\
\hline
\end{tabular}

*Recurrent or refractory ischemic pain or discomfort. 
tigators concluded that IMA may be a useful marker for the identification of ACS.

As these studies indicate, there is accumulating evidence that IMA levels correlate with, if not quantitatively predict, cardiac ischemic events. However, to the best of our knowledge no studies have been reported that evaluate the diagnostic value of IMA with an exclusive focus on shortterm, serious cardiac outcomes in patients for whom IMA might add valuable additional information about need for admission to hospital. Our focus on the relevant population and the most relevant outcome events makes our results directly applicable to the decision faced by physicians seeing patients with potential ACS in the emergency department.

We explored the possibility of multiple IMA thresholds, but the number of events proved too few. We also explored different thresholds (including the $85 \mathrm{U} / \mathrm{mL}$ suggested in the manufacturer's package insert), but none produced results that differed appreciably from those of our initial choice, $80 \mathrm{U} / \mathrm{mL}$. Other researchers used cut-off points of $75,{ }^{7} 80^{8}$ and $85 \mathrm{U} / \mathrm{mL} .13,17$ Given that the $95 \% \mathrm{CI}$ of the LRs fell outside $0.2-10$ in only 1 instance, and in all cases included a value of 1 , our results suggest that IMA levels do not provide information useful in predicting which patients will suffer a serious cardiac outcome in the short term. These results attest to the necessity for thorough evaluation of new diagnostic tests before their dissemination.

This article has been peer reviewed.

From the Department of Emergency Medicine, Hamilton Health Sciences (Worster, Opie); the Department of Medicine (Worster, Devereaux, Guyatt), Clinical Epidemiology and Biostatistics (Worster, Devereaux, Heels-Ansdell, Guyatt, Mookadam) and Pathology and Molecular Medicine (Hill), McMaster University; and the Hamilton Regional Laboratory Medicine Program (Hill), Hamilton, Ont.

\begin{tabular}{|c|c|c|c|c|c|c|}
\hline \multirow{2}{*}{ IMA } & \multicolumn{2}{|c|}{ Serious cardiac outcome } & \multicolumn{2}{|l|}{ Totals } & Estimate & $95 \% \mathrm{Cl}$ \\
\hline & Yes & No & & Sensitivity & 0.70 & $0.49-0.84$ \\
\hline & & & & Specificity & 0.24 & $0.18-0.32$ \\
\hline$>000 / \mathrm{mL}$ & 16 & 115 & 131 & $\mathrm{LR}+$ & 0.92 & $0.69-1.22$ \\
\hline$\leq 80 \mathrm{U} / \mathrm{mL}$ & 7 & 37 & 44 & LR- & 1.25 & $0.63-2.46$ \\
\hline \multirow{2}{*}{$3 \mathbf{h}$} & \multicolumn{2}{|r|}{ Total } & \multicolumn{2}{|l|}{175} & & \\
\hline & Yes & No & & Sensitivity & 0.81 & $0.57-0.93$ \\
\hline \multirow{2}{*}{$>80 \mathrm{U} / \mathrm{mL}$} & \multirow{2}{*}{13} & \multirow{2}{*}{117} & \multirow{2}{*}{130} & Specificity & 0.11 & $0.06-0.17$ \\
\hline & & & & $\mathrm{LR}+$ & 0.91 & $0.71-1.16$ \\
\hline \multirow[t]{2}{*}{$\leq 80 \mathrm{U} / \mathrm{mL}$} & 3 & 14 & 17 & LR- & 1.75 & $0.56-5.45$ \\
\hline & \multicolumn{2}{|r|}{ Total } & 147 & & & \\
\hline $6 \mathrm{~h}$ & Yes & No & & Sensitivity & 0.92 & $0.67-0.99$ \\
\hline \multirow{2}{*}{$>80 \mathrm{U} / \mathrm{mL}$} & \multirow{2}{*}{12} & \multirow{2}{*}{96} & \multirow{2}{*}{108} & Specificity & 0.06 & $0.03-0.12$ \\
\hline & & & & $\mathrm{LR}+$ & 0.98 & $0.83-1.16$ \\
\hline \multirow[t]{2}{*}{$\leq 80 \mathrm{U} / \mathrm{mL}$} & 1 & 6 & 7 & LR- & 1.31 & $0.17-10.02$ \\
\hline & & Total & 115 & & & \\
\hline \multicolumn{7}{|c|}{ Maximum IMA values } \\
\hline & Yes & No & & Sensitivity & 0.92 & $0.74-0.98$ \\
\hline & & & & Specificity & 0.06 & $0.03-0.11$ \\
\hline$>80 \mathrm{U} / \mathrm{mL}$ & 22 & 152 & 174 & $\mathrm{LR}+$ & 0.98 & $0.86-1.11$ \\
\hline \multirow[t]{2}{*}{$\leq 80 \mathrm{U} / \mathrm{mL}$} & 2 & 10 & 12 & LR- & 1.35 & $0.32-5.79$ \\
\hline & & Total & 186 & & & \\
\hline
\end{tabular}

Fig. 2: Measures of diagnostic efficacy of IMA levels at each collection time. The last set of data shows the highest IMA values measured before the occurrence of an adverse event, considering all 3 collection times. $\mathrm{Cl}=$ confidence interval, IMA = ischemia-modified albumin, $L R+=$ likelihood ratio (positive) for a serious adverse event to occur, LR- = likelihood ratio (negative) for the non-occurrence of an adverse event. 
Competing interests: None declared for P.J. Devereaux, Dianne Heels-Ansdell, Gordon Guyatt, John Opie and Farouk Mookadam. Andrew Worster and Stephen Hill have received partial coverage of expenses from Ischemia Technologies, Denver, Colo., to attend a meeting at the company office to discuss study plans.

Contributors: Study leader Andrew Worster led the group in protocol development, trial performance and data analysis and interpretation, and wrote the first draft of this report. Stephen Hill, also a principal investigator, led the laboratory component of the trial and was active in assembling and validating the data and preparing the manuscript. As well as taking part in protocol development, P.J. Devereaux organized the adjudication process, took part in data analysis and interpretation, and contributed to manuscript preparation. Statistician Dianne Heels-Ansdell created the database, led the acquisition of data and performed the statistical analysis. Gordon Guyatt not only helped in protocol development, data analysis and interpretation and manuscript preparation, he also provided expert guidance at all stages. John Opie took part in protocol development, helped perform the trial in the emergency department, and adjudicated the clinical data. Farouk Mookadam also helped develop the study protocol, especially during the early stages.

Acknowledgements: P.J. Devereaux is supported by a Senior Research Fellowship Award from the Canadian Institutes of Health Research. We also appreciate the outstanding work of Kim Elliot, who coordinated this study.

This study was supported by a research grant from Ischemia Technologies, Denver, Colo.

\section{References}

1. Bar-Or D, Lau E, Winkler JV. A novel assay for cobalt-albumin binding and its potential as a marker for myocardial ischemia - a preliminary report. 7 Emerg Med 2000;19(4):311-5.

2. McCord JM. Oxygen-derived free radicals in post-ischemic tissue injury [review]. N Engl 7 Med 1985;312(3):159-63.

3. Cobbe SM, Poole-Wilson PA. The time of onset and severity of acidosis in myocardial ischaemia. 7 Mol Cell Cardiol 1980;12(8):745-60

4. Berenshtein E, Mayer B, Goldberg C, Kitrossky N, Chevion M. Patterns of mobilization of copper and iron following myocardial ischemia: possible predictive criteria for tissue injury. 7 Mol Cell Cardiol 1997;29(11):3025-34.

5. Bar-Or D, Winkler JV, Vanbenthuysen K, Harris L, Lau E, Hetzel FW. Reduced albumin-cobalt binding with transient myocardial ischemia after elective percutaneous transluminal coronary angioplasty: a preliminary comparison to creatine kinase-MB, myoglobin, and troponin I. Am Heart 7 2001;141 (6):985-91.

6. Bar-Or D, Curtis G, Rao N, Bampos N, Lau E. Characterization of the $\mathrm{Co}^{2+}$ and $\mathrm{Ni}^{2+}$ binding amino-acid residues of the $\mathrm{N}$-terminus of human albumin: an insight into the mechanism of a new assay for myocardial ischemia. Eur 7 Biochem 2001;268(1):42-7.

7. Christenson RH, Duh SH, Sanhai WR, Wu AH, Holtman V, Painter P, et al. Characteristics of an albumin cobalt binding test for assessment of acute coronary syndrome patients: a multicenter study. Clin Chem 2001;47(3):464-70.

8. Wu AH, Morris DL, Fletcher DR, Apple FS, Christenson RH, Painter PC. Analysis of the albumin cobalt binding (ACB) test as an adjunct to cardiac troponin I for the early detection of acute myocardial infarction. Cardiovasc Toxicol 2001;1(2):147-51.

9. Jaeschke R, Guyatt G, Lijmer J. Diagnostic tests. In: Guyatt G, Rennie D.The Users' Guides to the Medical Literature: a manual for evidence-based clinical practice. Chicago: AMA Publications; 2002. p. 121-40.

10. Hill SA, Devereaux PJ, Griffith L, Opie J, McQueen MJ, Panju A, et al. Can troponin I measurement predict short-term serious cardiac outcomes in patients presenting to the emergency department with possible acute coronary syndrome? Can 7 Emerg Med 2004;6(1):223-30.

11. Rao AC, Naeem N, John C, Collinson PO, Canepa-Anson R, Joseph SP. Direct current cardioversion does not cause cardiac damage: evidence from cardiac troponin T estimation. Heart 1998;80:229-30.

12. Roy D, Quiles J, Sinha M, Aldama G, Gaze D, Kaski JC. Effect of directcurrent cardioversion on ischemia-modified albumin levels in patients with atrial fibrillation. Am 7 Cardiol 2004;93(3):366-8

13. Sinha MK, Roy D, Gaze DC, Collinson PO, Kaski JC. Role of "ischemia modified albumin", a new biochemical marker of myocardial ischaemia, in the early diagnosis of acute coronary syndromes. Emerg Med 7 2004;21(1):29-34.

14. Quiles J, Roy D, Gaze D, Garrido IP, Avanzas P, Sinha M, et al. Relation of ischemia-modified albumin (IMA) levels following elective angioplasty for stable angina pectoris to duration of balloon-induced myocardial ischemia. Am 7 Cardiol 2003;92(3):322-4.

15. Sinha MK, Gaze DC, Tippins JR, Collinson PO, Kaski JC. Ischemia modified albumin is a sensitive marker of myocardial ischemia after percutaneous coronary intervention. Circulation 2003;107(19):2403-5.

16. Garrido IP, Roy D, Calvino R, Vazquez-Rodriguez JM, Aldama G, CosinSales J, et al. Comparison of ischemia-modified albumin levels in patients undergoing percutaneous coronary intervention for unstable angina pectoris with versus without coronary collaterals. Am 7 Cardiol 2004;93(1):88-90.

17. Roy D, Quiles J, Aldama G, Sinha M, Avanzas P, Arroyo-Espliguero R, et al Ischemia modified albumin for the assessment of patients presenting to the emergency department with acute chest pain but normal or non-diagnostic 12-lead electrocardiograms and negative cardiac troponin T. Int 7 Cardiol 2004;97(2):297-301.

Correspondence to: Dr. Stephen Hill, Laboratory Medicine, Hamilton General Hospital, 237 Barton St. E, Hamilton, ON L8L 2X2; fax 905 577-8028; hillstev@hhsc.ca

\section{P M I S C H E D U L E}

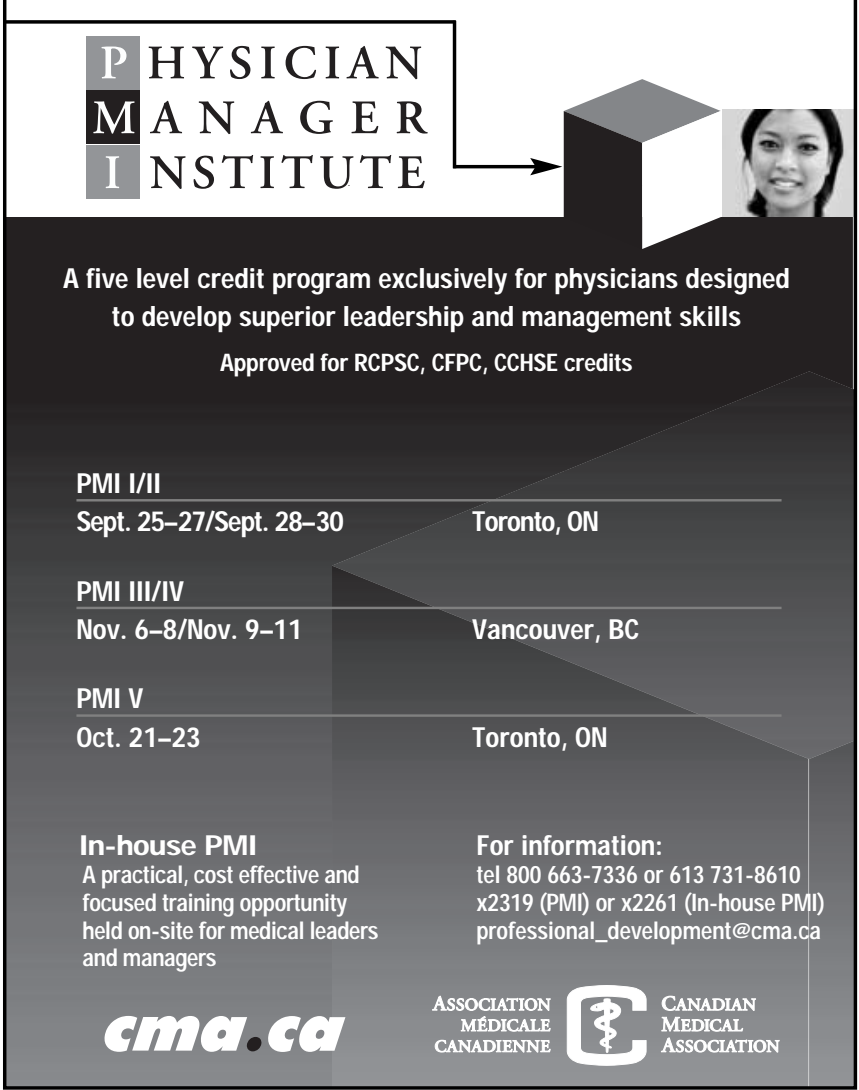

